\title{
A Seamless Broadband PLC-VLC Transmission: Performance Evaluation and Dimensioning
}

\author{
Yara Yaacoub, F. Nouvel, S. Haese and J.-Y Baudais \\ Univ Rennes, INSA Rennes, CNRS, IETR-UMR 6164 \\ F-35000 Rennes, France \\ email: yara.yaacoub@insa-rennes.fr
}

\begin{abstract}
As the LED lamps are naturally connected to the powerline, the integration of powerline communication (PLC) and visible light communication (VLC) seems to be trivial. In fact, cascading VLC to PLC is not very simple especially because their channels are not designed for communication purposes. The PLC and VLC channels suffer from severe attenuation with increasing frequency. Thus, the cascading of these two channels will lead to a very limited bandwidth which can complicate the PLC-VLC integration. Few experimental studies are carried out to prove the feasibility of PLC-VLC integration, particularly those based on the amplify and forward (AF) relay. Therefore, PLC-VLC broadband transmission is demonstrated in this paper without making any changes to the transmitted PLC signal before traveling through the optical system. A theoretical study is also carried out to design LED luminaires capable of providing both lighting and communication. This study extrapolates the experimental results of the small-scale PLC-VLC testbed to design a PLC-VLC system for typical indoor applications.

Index Terms-Powerline communication,visible light communication, powerline and visible light communication integration, OFDM, HomePlug AV.
\end{abstract}

\section{INTRODUCTION}

Recently, the demand for high-speed data is greatly increasing, resulting in spectrum congestion in RF wireless technologies. One of the best solutions is to migrate wireless communication to the visible spectrum. After the rapid development of InGaN-based LEDs in the lighting industry, researchers are increasingly drawn to study the ability to transmit communication signals through LED bulbs firstly dedicated for indoor lighting. However, VLC cannot be operated on its own. It is a last mile communication system that must be connected to a backbone. In fact, PLC acts as a natural backbone for the VLC, as the LED light has to be powered using the powerline network. In this case, the PLC system acts similar to Ethernet towards Wifi. On the other hand, PLC is a very mature technology and it is used in many applications such as indoor communication, vehicular communication, smart meters, etc. But it should be noted that just like LED luminary, PLC networks were not designed to transport communication signals. Many problems are encountered by PLC designers due to the inability of this medium to support high frequency signals. Fortunately, modulation techniques such as orthogonal frequency division multiplexing (OFDM) are being developed to overcome multipath and frequency selective channels like PLC channels.
The integration of PLC-VLC system is firstly proposed in [1] using single carrier binary phase shift keying modulation. Then, in [2] narrowband OFDM is deployed in PLC-VLC system. In spite of the existence of many studies to optimize the integration of these two systems [3], [4], the number of experimental trials is quite limited. In [5], a hybrid broadband AF PLC-VLC system is proposed using OFDM modulation for localization and communication within hospitals. In [6], video was transmitted through a PLC-VLC AF system using an array of three LED lamps. For [5], [6] the PLC-VLC system bandwidth is limited to $8 \mathrm{MHz}$ located from 2 to $10 \mathrm{MHz}$ because a limited frequency response LED lamp used (white LED with a phosphor coating). In [7], a duplex PLC-VLC system is proposed based on the decode and forward approach. The data rate achieved is $5 \mathrm{Mbit} / \mathrm{s}$ and it can be extended to $30 \mathrm{Mbit} / \mathrm{s}$. Thus, to the authors' knowledge, there is no report up to date on the feasibility of transmitting HomePlug AV (HPAV) data directly into the optical system without making any modification to the PLC signal before being transmitted by the VLC system.

In this paper, a PLC-VLC broadband system is proposed to test the possibility of transmitting a HPAV signal directly into the VLC system. This system uses only the red LED of an RGBW LED module to extend the modulation bandwidth as much as possible. User Data Protocol (UDP) packet transmissions are performed to evaluate our testbed performance. In addition, a theoretical study is performed taking into account the number of LEDs that should be used, the distance between the optical transmitter and the receiver, and the position of the receiver in relation to the transmitter.

The remainder of this paper is organized as follow: In Section II, the proposed PLC-VLC system is dissected and the choice of each component is discussed. A brief explanation of the HPAV standard is also given as well as a small overview of the adopted VLC system. In Section III, an online UDP packets transmission is demonstrated using our testbed. Several throughput measurement attempts are presented varying at each time the distance between the optical transmitter and the receiver followed by a throughput comparison between PLC and PLC-VLC systems. In Section IV, a theoretical study is carried out to dimension the PLC-VLC system for typical indoor application. This study is based on the calculation of the required number of LEDs that should be used to ensure the maximum data rate and the desired lighting characteristics. 
Table I: HomePlug AV parameters.

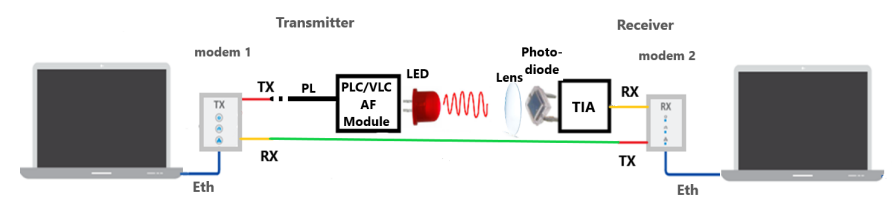

Figure 1: Diagram of the hybrid PLC-VLC system.

In addition to an optical power simulation in function of the receiver position relative to the optical emitter to highlight the effect of the receiver mobility on the optical communication performance. Finally, a conclusion summarizing the points covered in this paper is presented in Section V.

\section{SYSTEM DESCRIPTION}

In this section, a simple hybrid PLC-VLC system is proposed in order to investigate the possibility of transmitting a signal of an already existing broadband powerline standard through VLC. In this study, the proposed hybrid PLCVLC system makes use of a commercial PLC modem which transmits a HPAV signal. The signal transmitted by the first PLC modem (modem 1) is injected into the LED via the AF PLC-VLC module (see Fig. 1). In this hybrid AF PLC-VLC transmitter, the PLC signal is absolutely not demodulated and remodulated for the VLC part [8]. The LED retransmits the original PLC signal to the optical receiver consisting of a photodiode and a transimpedance amplifier (TIA). In order to perform fast real time decoding, the received optical signal is sent back to the second powerline modem (modem 2), (see Fig. 1) which plays the role of the decoder. It should be mentioned herein, that the powerline modems are halfduplex, so downlink communication should also be fulfilled. Note that, as this paper focuses on the PLC-VLC simplex communication, the downlink is simply connected using a wire.

The HPAV standard is specified for fast in-home power line networking. It features an advanced physical bandwidth operating in the $2-30 \mathrm{MHz}$ band. This band is the most compatible with our VLC system as it will be shown in the next paragraph. The HPAV media access control layer defines operation using carrier sense multiple access with collision avoidance (CSMA/CA), but it also includes time domain multiple access (TDMA) with a central coordinator as well as a hybrid CSMA/TDMA mode. The physical layer uses OFDM modulation technique with an adaptive bit-loading scheme that enable adaptation to the powerline channel and impulsive noise, as presented in Table I [9]. In our test bed, we use the dLAN 200 AV PLC modems from Devolo [10] because these modems adopt HPAV standard.

Concerning the VLC part, it is composed of an optical transmitter and receiver (see Fig.2). The optical transmitter consists of a bias tee circuit and a LED. The bias tee is a

\begin{tabular}{c|c}
\hline Parameter & Value \\
\hline Scrambler & Yes \\
FEC & Turbo code \\
Interleaver & Yes \\
Mapper & QAM 2 to 1024 \\
Modulation & Windowed-OFDM \\
IFFT/FFT size & 3072 \\
Number of carriers & 1536 \\
Sampling frequency & $75 \mathrm{MHz}$ \\
Bandwidth & $2-28 \mathrm{MHz}$ \\
Inter-carriers space & $24.414 \mathrm{kHz}$ \\
Symbol time & $40.96 \mu \mathrm{s}$ \\
Guard interval & $5.56 \mu$ s or $7.56 \mu$ s or $47.12 \mu \mathrm{s}$ \\
MAC layer protocol & Hybrid: CSMA/CA \& TDMA \\
\hline \multicolumn{2}{|}{}
\end{tabular}

three-port network. The low-frequency port is used to define the polarization, whereas the high-frequency port passes the data signals but blocks bias levels, and the combo port is connected to the LED, which can provide both polarization and data signals. In order to use the VLC LEDs for lighting, white light should be adopted. To generate a white light, there are two main technologies in lighting industry: the combination of a red, green, and blue LEDs or a blue LED with a phosphor coat. Moving to the optical receiver, it consists of a photodiode, a TIA, high pass filter (HPF), and a voltage amplifier (VA). The photodiode converts the received optical power into an electrical current. The TIA amplifies and transforms this current into voltage. The HPF eliminate the DC component. The VA further amplifies the recovered signal. The used bias tee is designed with a large bandwidth $(20 \mathrm{kHz}-100 \mathrm{MHz}$ ) and an RGBW LED (Luxeon multicolor $2.5 \mathrm{~W}$ [11]) is chosen in this paper as the phosphor coat can limit the LED bandwidth. Moreover, measurements performed on the chosen RGBW modules show that the red LED has the largest bandwidth $(20 \mathrm{MHz})$ [12]. For this reason and to simplify the presentation of the study, the red LED is only used to transmit the HPAV signal. Note that the use of the green, blue and white LEDs is possible for data transmission but they do not increase the transmission bandwidth. Thus, they can be used to create the white light color. The chosen photodiode in our study should have a large bandwidth and, conversely, a large active area in order to receive the maximum possible of the optical power. These two conditions cannot be fulfilled simultaneously because by increasing the surface area of the photodiode, the equivalence capacitor will increase, thus limiting its bandwidth. A compromise is found in the SFH2400 photodiode [13]. The TIA, the HPF and the VA are realized in a way to provide a great amplification gain, a large bandwidth, and low noise. The circuit design is based on the method described in [12] which allows the calculation of the generated photo-current as a function of the transmitted current. The VLC part parameters are listed in Table II.

To evaluate the possible data rate of the PLC-VLC system, UDP packets of 1470 bytes are sent from a computer connected to modem 1 (client) through an Ethernet cable during $40 \mathrm{~s}$. Another computer connected to modem 2 (server) 


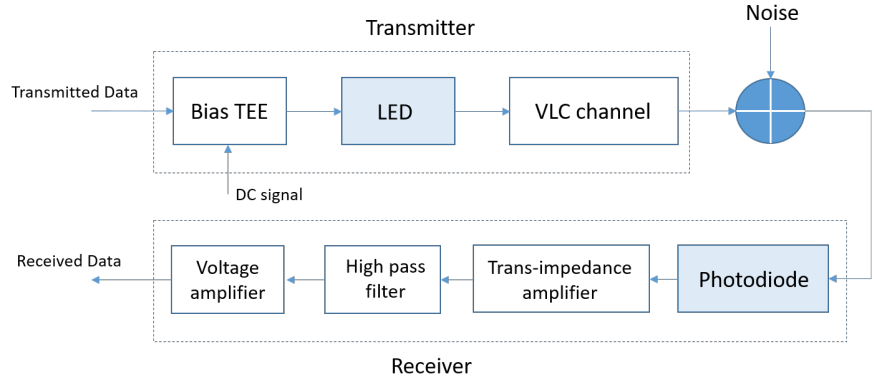

Figure 2: Bloc diagram of the VLC part.

Table II: VLC system parameters.

\begin{tabular}{c|c}
\hline Index & Value \\
\hline LED luminous flux & $18 \mathrm{~lm}$ \\
Dominant wavelength of the LED & $618 \mathrm{~nm}$ \\
LED Half intensity beam angle & $85^{\circ}$ \\
Input current & $120 \mathrm{~mA}$ \\
Bias-Tee bandwidth & $20 \mathrm{kHz}-100 \mathrm{MHz}$ \\
Photodiode sensitivity & $0.44 \mathrm{~A} / \mathrm{W}$ \\
Photodiode active area & $1 \mathrm{~mm}^{2}$ \\
TIA, HPF and VA total gain & $0.2 \mathrm{M} \Omega$ \\
TIA, HPF and VA total bandwidth & $70 \mathrm{MHz}$ \\
\hline
\end{tabular}

receives the packets transmitted through our hybrid system. Since the PLC-VLC system is simplex, UDP packets are chosen to be transmitted because no acknowledgment packet is required to be sent on the downlink as for Transmission Control Protocol.

\section{System EVALUATION}

In this section, the feasibility of the proposed PLC-VLC system is proven by experiments. Fig. 3 shows the maximum throughput as a function of the offered load and the distance between the LED and the optical receiver. Obviously, the greatest value of the maximum throughput is reached (between $58 \mathrm{Mbit} / \mathrm{s}$ and $68 \mathrm{Mbit} / \mathrm{s}$ ) for distances less than $40 \mathrm{~cm}$. Beyond this distance, the maximum throughput begins to decrease until it reaches $17.06 \mathrm{Mbps}$ at a distance of $85 \mathrm{~cm}$. It should be noted that the PLC topology used in the testbed is very simple (only two nodes) and has almost no effect on the signal.

In order to illustrate the impact of integrating the VLC system into the PLC system, a UDP throughput comparison is carried out between the PLC system and the PLC-VLC system as a function of the transmitted power by the modem 1 . To be able to vary the power of the signal transmitted by the modem 1, a variable gain power attenuator is placed at modem 1's output. In the PLC-VLC system, the distance between the LED and the optical receiver is fixed at $10 \mathrm{~cm}$. As shown in Fig. 4, the maximum PLC throughput is almost constant (maximum) for these power values. However, in the PLC-VLC system, additional power is required to maintain the maximum throughput of $66 \mathrm{Mbit} / \mathrm{s}$. The reason behind the need for additional power to maintain maximum throughput in the case of PLC-VLC system, despite the fact that PLC modems are designed to tolerate low power values since the

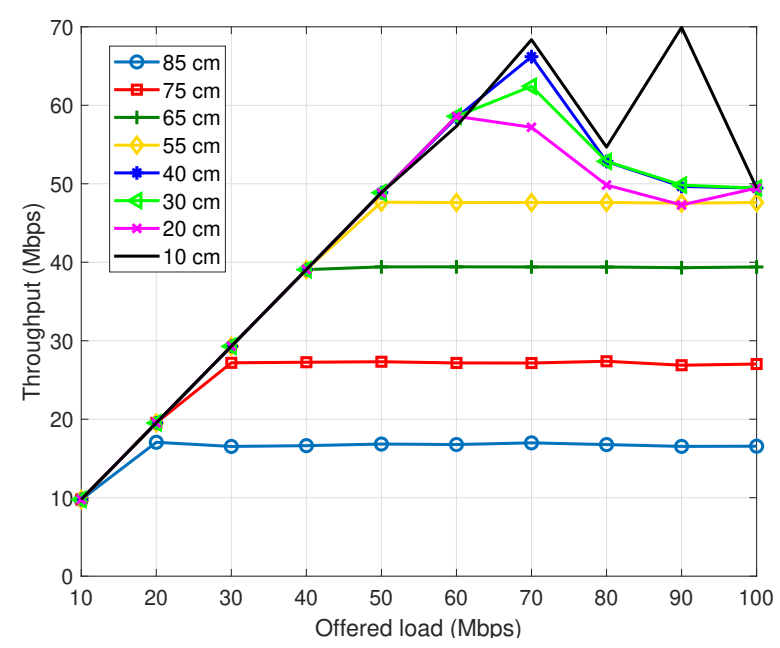

Figure 3: UDP Throughput versus the offered load for different distance between the LED and the optical receiver.

PLC signal is exposed to high attenuation when it travels over Powerline networks, is that the signal to noise ratio (SNR) in the case of the PLC-VLC system is much lower than that of the PLC system due to the addition of VLC noise and the limitation of the LED bandwidth .

To further highlight the effect of limited LED bandwidth and VLC noise, the UDP throughput of both systems (full PLC and PLC-VLC) are compared when modem 2 receives the same amount of power. Considering the case where the PLC-VLC system transmits a signal of power $-8 \mathrm{dBm}$, modem 2 receives a power of $-20 \mathrm{dBm}$. In this case, the maximum throughput reached is about $27 \mathrm{Mb} / \mathrm{s}$. However in the case of the full PLC system, when modem 2 receives a power of $-20 \mathrm{dBm}$ the maximum throughout achieved is approximately $67 \mathrm{Mb} / \mathrm{s}$. Thus, even if modem 2 receives the same amount of power in both systems, the SNR in the case of PLC-VLC is lower than that of the PLC system.

\section{OPTICAL SYSTEM DIMENSIONING}

As seen in the previous section, $40 \mathrm{~cm}$ is the maximum distance between the LED and the photodiode to obtain the maximum throughput between PLC-VLC modems (66 Mbits). The measurement shows that the electrical power received at this distance is $-7 \mathrm{dBm}$. Thus, it can be predicted that this value is the minimum power value which guarantees the maximum throughput of PLC-VLC modems. In order to increase the distance and cover a larger surface area, multiple LEDs should be used. Therefore, in this section, a theoretical analysis is done to find the minimum number of red LEDs needed to achieve the maximum throughput.

The received electrical power $P_{r}$ is inversely proportional to the square of the distance separating the LED from the photodiode as shown by the following expression [14]: 


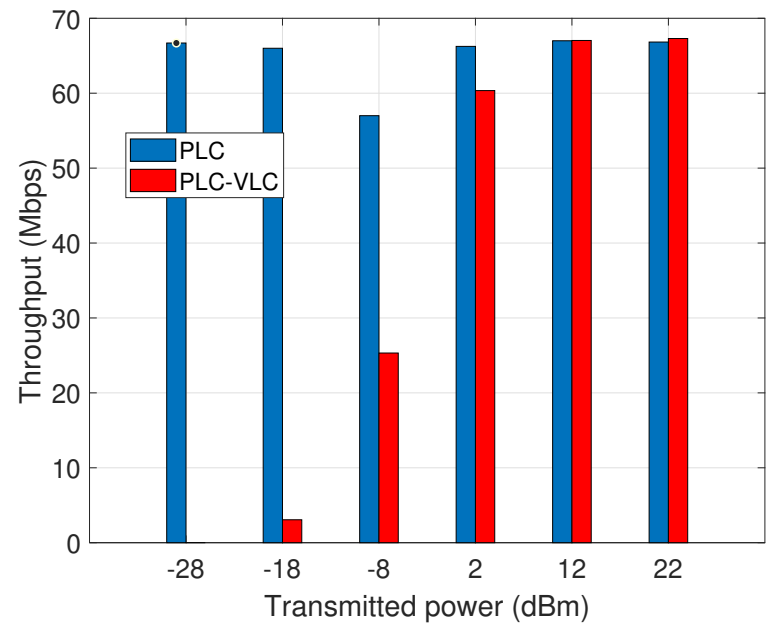

Figure 4: UDP Throughput comparison between PLC and PLC-VLC system in function of the Transmitted power.

$$
\begin{aligned}
P_{r}= & H_{O / E} \sum_{n=1}^{N_{\mathrm{LED}}} \frac{(m+1) A \cos ^{m}(\Phi) \cos (\psi) G}{2 \pi D^{2}} H_{E / O} P_{t r} \\
& \psi<\psi_{\mathrm{FOV}}
\end{aligned}
$$

where

$$
D=\sqrt{x^{2}+y^{2}+z^{2}}
$$

and

$$
m=\frac{\ln (2)}{\ln \left(\cos \left(\Phi_{1 / 2}\right)\right.}
$$

and $N_{\text {LED }}$ is the number of used LEDs, $m$ is the Lambertian index which is given by the semi-angle at half irradiance of a LED $\Phi_{1 / 2}, A$ is the active area of the photodiode, $\Phi$ and $\psi$ are the angles of irradiance and incidence respectively, $D$ is the distance between the LED and the photodiode, $\psi_{\mathrm{FOV}}$ is the irradiance angle of the field of vision of the receiver, $G$ is the gain of the lens that concentrates the light to the photodiode, $P_{t r}$ is the transmitted electrical power, $H_{O / E}$ and $H_{E / O}$ are the electrical to optical and the optical to electrical conversions respectively which are calculated according to the expression developed in [12], and $(x, y, z)$ are the spatial coordinates of the optical receiver considering the optical transmitter as the origin. Maintaining sufficient received power while increasing the distance requires an increase in the transmitted light power, that is to say more LED or increasing the transmitted power per LED which may not be always possible because the transmitted power is limited to a certain level to avoid saturation of the LED and eventually distorting the signal. The required number of LED is calculated according to equation (1). In the simulation, the electrical power of the transmitted signal is fixed to $23.53 \mathrm{dBm}$ which is the maximum power that the red LED can withstand [11]. Based on the parameters of our VLC system, the minimum number of LEDs needed as a function of the distance $D$ is calculated as shown in Fig. 5. Considering a typical room of $3 \mathrm{~m}$ in height, the distance between the LED and a receiver located on a work area for work purposes, typically $0.75 \mathrm{~m}$ above the ground, is approximately $2.25 \mathrm{~m}$. At this distance of $2.25 \mathrm{~m}$, the minimum number of red LED is 60 (see Fig. 5). This number should be compared to the number of LED required to illuminate the room to validate that no additional LED are needed to maintain good communication performance. Based on the Deutsche Institut fur Normung (DIN) standard, the required illuminance of a normal office room is 500 lux [15]. The total luminous flux can be calculated as a function of the horizontal illuminance $\left(E_{h}\right)$ by the following expression [16]:

$$
E_{h}=\sum_{n=1}^{N_{\mathrm{LED}}} \frac{(m+1) A \cos ^{m}(\Phi) \cos (\psi)}{2 \pi D^{2}} \Phi_{n}
$$

where $\Phi_{n}$ is the luminous flux of the $n$th LED. The target luminous flux at a distance of $2.25 \mathrm{~m}$ should be $7078 \mathrm{~lm}$. This value cannot be achieved by using only a single RGBW module because their LED are very small. As mentioned in [11], the red, green, blue and white LED of the module provide respectively $18,38,12$ and $53 \mathrm{~lm}$ which can't suffice for lighting the room. Thus, an array of multi-LED should be used. The minimum number of red, green, blue and white LEDs needed to get a white light of $7078 \mathrm{~lm}$ with a color temperature of $4000 \mathrm{~K}$ (cool white) are derived making use of ColorCalculator software developed by OSRAM [17] via the following procedure: at first the target luminous flux, the luminous flux and the wavelength of each colored LED should be known. Then, using the ColorCalculator software, the chromatic coordinates of each of the LED color and the target light color is extracted from the CIE 1931 xy color space (see Fig. 6). By exhaustive trials using the same software, the total luminous flux of each of the four colors is found such that the sum of the luminous flux of these four colors is equal to the target luminous flux and that their proportions reach the chromatic coordinates of the desired color as shown in Fig. 6. Finally, the number of LED for each color is obtained by dividing the total required luminous flux by the luminous flux emitted by a single LED. Table III shows the total luminous flux and the number of LED needed for each color. As seen in table III, the minimum number of red LED needed for lighting is $71 \mathrm{LED}$, which is greater than that required for data transmission which is 60 LED. Hence, this total number of LED needed for lighting can easily transmit the UDP packets at a rate of $66 \mathrm{Mb} / \mathrm{s}$.

Table III: Design of a $4000 \mathrm{~K}$ white light system using red, green and blue LED from Luxeon module [17].

\begin{tabular}{c|c|c|c|c}
\hline LED & Red & Green & Blue & White \\
\hline Required total luminous flux $(\mathrm{lm})$ & 1278 & 2584 & 696 & 3127 \\
\hline Required number of LED & 71 & 68 & 58 & 59 \\
\hline
\end{tabular}

Furthermore, the receiver may be mobile within the room. Thus, it is difficult to receive the same amount of power in any position of the room. Hence, a second theoretical study is 
carried out to show the effect of the position of the receiver with respect to the optical transmitter. This study is based on the expression developed in [12] which allows a simple calculation of the electrical power received as a function of the LED channel, taking into account the optical, electrical and frequency behavior of the VLC system. Specifically, the received electrical power is calculated based on the position of the receiver relative to the optical transmitter in a typical $5 \times 5 \times 3 \mathrm{~m}^{3}$ office. The optical emitter situated at $(0,0,0)$ is considered to have an array of 64 LEDs separated by $1 \mathrm{~cm}$ from each other. As shown in Fig. 7, the power attenuation increases as the receiver moves away from the LED until it reaches $-26.81 \mathrm{~dB}$ at the corners of the room. So, a LED distribution method should be figured out in order to ensure equal power coverage throughout the room.

\section{CONCLUSION}

In this paper, a broadband PLC-VLC system is proposed. The direct transmission of an already existing broadband PLC standard is demonstrated by experimental tests. Despite the simplicity of our system which does not make any modification to the PLC signal before being transmitted by the VLC system, the maximum throughput of $66 \mathrm{Mbit} / \mathrm{s}$ is reached. The choice of the component that constitute our system is also explained in this paper. In addition, a comparison between the performance of PLC and PLC-VLC systems is presented to show the effect of integrating the VLC system with the PLC. Finally, optical system dimensioning is carried out in order to extrapolate our small scale PLC-VLC testbed to a typical indoor application. A comparison is also made between the number of red LED needed for lighting and the number needed for data transmission to ensure that adding VLC to a lighting system will not require an additional LEDs.

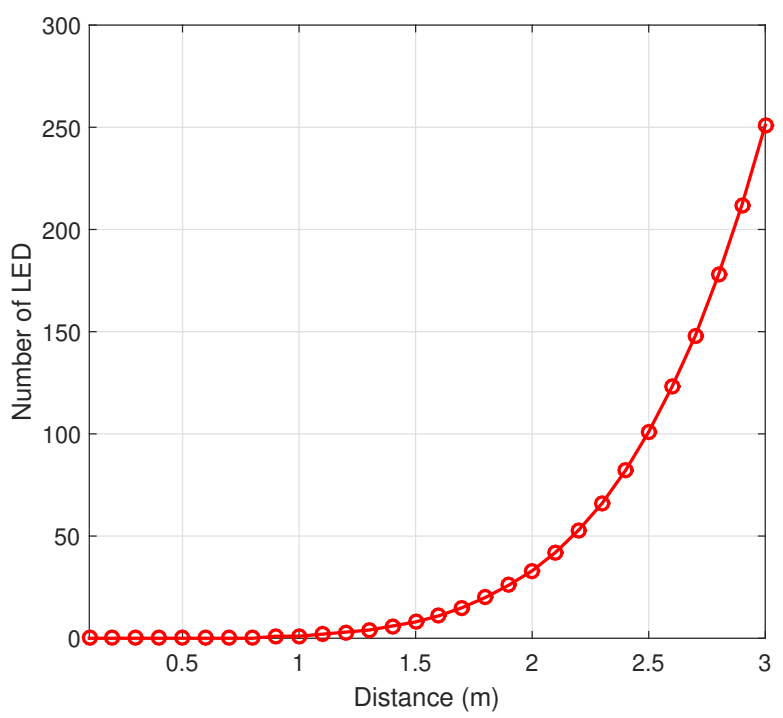

Figure 5: Number of LED needed to receive $-7 \mathrm{dBm}$ signal as a function of the distance between the LED and the photodiode.

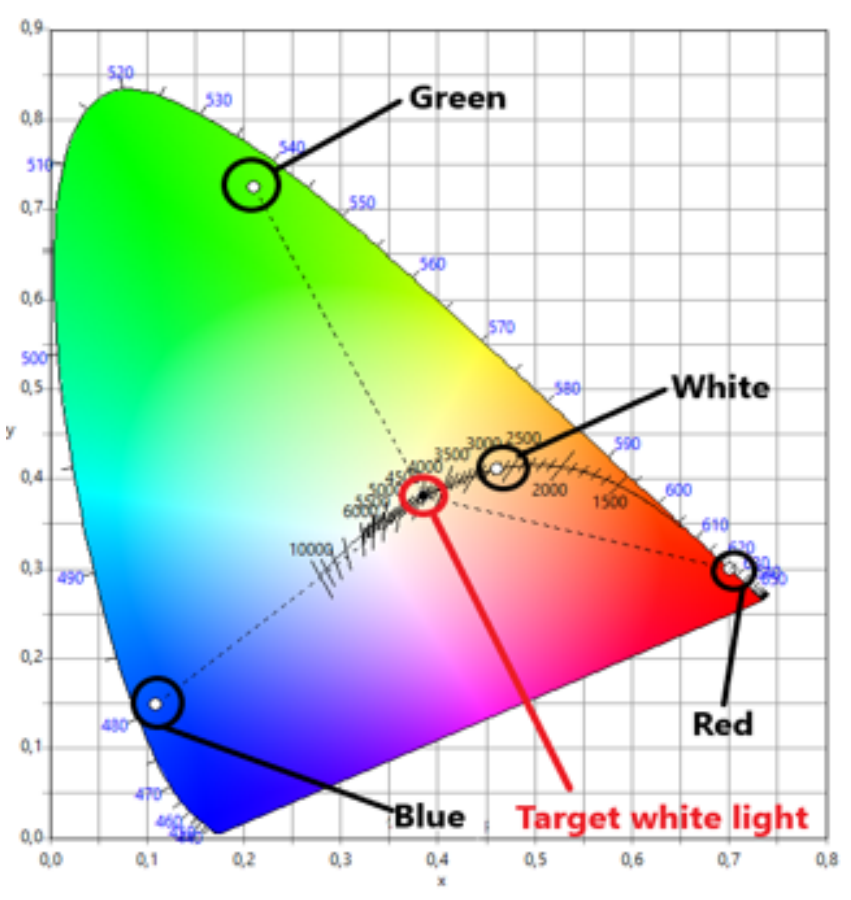

Figure 6: CIE 1931 xy color space with the red, green, blue and white LED chromatic coordinates and the target white light chromatic coordinates [17].

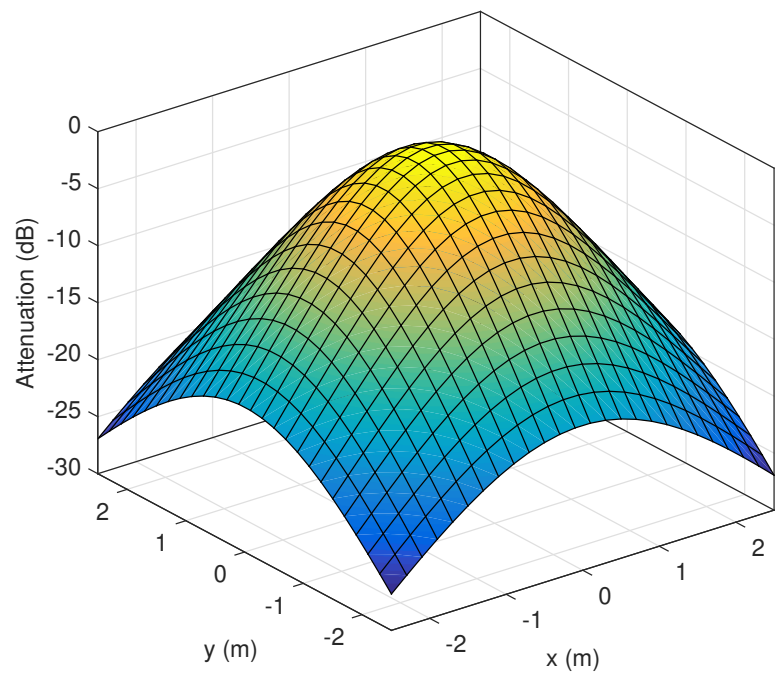

Figure 7: Received power attenuation as a function of the receiver position in a $5 \times 5 \times 3 \mathrm{~m}^{3}$ office. 


\section{ACKNOWLEDGMENT}

This work has been supported by the Pôle d'excellence cyber under the project "PEC-Region Bretagne PLC-VLC $\mathrm{n}^{\circ}$ 1208 ".

\section{REFERENCES}

[1] T. Komine and M. Nakagawa, "Integrated system of white LED visible-light communication and powerline communication", IEEE Transactions on Consumer Electronics, vol. 49, no. 1, pp. 71-79, 2003.

[2] T. Komine, S. Haruyama, and M. Nakagawa, "Performance evaluation of narrowband ofdm on integrated system of power line communication and visible light wireless communication", in International Symposium on Wireless Pervasive Computing, 2006, 6 pp.-6.

[3] W. Gheth, K. M. Rabie, B. Adebisi, et al., "Performance analysis of integrated power-line/visible-light communication systems with af relaying", in IEEE Global Communications Conference, 2018, pp. 1-6.

[4] S. Baig, H. Muhammad Asif, T. Umer, et al., "High data rate discrete wavelet transform-based PLC-VLC design for $5 \mathrm{~g}$ communication systems", IEEE Access, vol. 6, pp. 52 490-52 499, 2018.

[5] W. Ding, F. Yang, H. Yang, et al., "A hybrid power line and visible light communication system for indoor hospital applications", Computers in Industry, vol. 68, pp. 170-178, 2015.

[6] Y. Yan, W. Ding, H. Yang, et al., "The video transmission platform for the PLC and VLC integrated system", in IEEE International Symposium on Broadband Multimedia Systems and Broadcasting, 2015, pp. 1-5.

[7] J. Song, S. Liu, G. Zhou, et al., "A cost-effective approach for ubiquitous broadband access based on hybrid PLC-VLC system", in IEEE International Symposium on Circuits and Systems, 2016, pp. 2815-2818.

[8] M. S. A. Mossaad, S. Hranilovic, and L. Lampe, "Amplify-and-forward integration of power line and visible light communications", in 2015 IEEE Global Conference on Signal and Information Processing (GlobalSIP), 2015, pp. 1322-1326.

[9] S. Galli, M. Koch, H. A. Latchman, et al., "Industrial and international standards on PLC-based networking technologies", in Power Line Communications. John Wiley \& Sons, Ltd, 2010, ch. 7, pp. 363-412.

[10] devolo AG, Germany, Nov. 2006.

[11] Luxeon multicolor module 2.5W, DS244, Lumileds, Apr. 2020.

[12] Y. Yaacoub, F. Nouvel, S. Haese, et al., "VLC modelization for VLC-PLC system: Evaluation of optical, electrical, and frequency behavior", in IEEE International Symposium on Circuits and Systems, 2021, pp. 1-5.

[13] Silicon PIN photodiode sfh2400-z, 81521, OSRAM, Apr. 2016.
[14] T. Komine and M. Nakagawa, "Fundamental analysis for visible-light communication system using LED lights", IEEE Transactions on Consumer Electronics, vol. 50, no. 1, pp. 100-107, 2004.

[15] Artificial lighting; recommended values for lighting parameters for indoor and outdoor workspaces, DIN, Sep. 1990.

[16] A. Jabban, S. Haese, and M. Helard, "Theoretical and experimental optimization of DMT-based visible light communication under lighting constraints", EURASIP Journal on Wireless Communications and Networking, vol. 2020, Dec. 2020.

[17] Colorcalculator user guide, OSRAM Sylvania, Inc., Sep. 2019. 